\title{
Time Resolved Optical Studies of InGaN Layers Grown on LGO
}

Maurice Cheung, Gon Namkoong ${ }^{1}$, Madalina Furis, Fei Chen, Alexander. N. Cartwright, W. Alan Doolittle ${ }^{1}$ and April Brown ${ }^{2}$, University at Buffalo, State University of New York, Buffalo, NY 14260, U.S.A.

${ }^{1}$ Geogia Institute of Technology, Atlanta, GA

${ }^{2}$ Duke University, Durham, NC

\section{ABSTRACT}

Radiative recombination processes in bulk InGaN grown by molecular beam epitaxy (MBE) on lithium gallate ( $\mathrm{LGO}$ or $\mathrm{LiGaO}_{2}$ ) substrates were investigated using microscopic PL and time-resolved photoluminescence (TRPL). The improved structural quality resulting from a better lattice match of the LGO substrate to III-V nitride materials simplifies these investigations because well-defined composition phases can be analyzed for both homogeneous and phased separated InGaN samples. Epilayers of InGaN intentionally grown with and without indium segregation were studied. X-ray diffraction measurements showed that the homogeneous epilayer was high quality $\operatorname{In}_{0.208} \mathrm{Ga}_{0.702} \mathrm{~N}$ and the segregated epilayer exhibited peaks corresponding to both $\mathrm{In}_{0.289} \mathrm{Ga}_{0.711} \mathrm{~N}$ and $\mathrm{In}_{0.443} \mathrm{Ga}_{0.557} \mathrm{~N}$ indicating the presence of higher In concentration regions in this sample. Spatially resolved photoluminescence spectra confirm the existence of these regions. The photoluminescence intensity decay is non-exponential for both samples and a stretched exponential fit to the decay data confirms the existence of local potential fluctuations in which carriers are localized before recombination.

\section{INTRODUCTION}

InGaN-based emitters are widely used for commercial high-brightness light emitting diodes (LED) from UV to amber [1,2]. There have been numerous reports on the improvement of efficiency in these LED devices due to In-segregation [3-7]. The current theory suggests that the In-rich regions existing inside InGaN act as localization centers, which trap the carriers injected into the system. The probability of non-radiative recombination for a carrier localized in a potential fluctuation is significantly reduced $[4,6]$ and the excitons formed in the vicinity of the localization centers have greater oscillator strength [6]. As a result, the optical efficiency of the LED is greatly improved.

Most of the commercially available InGaN emitters consist of a multiple quantum well (MQW) heterostructures, which are characterized by emission efficiency that depends not only on the In-segregation but also on the built-in electric fields present as a result of the piezoelectric polarization [8-10]. Here, we have studied the changes induced by In-segregation in an InGaN epilayer grown by Molecular Beam Epitaxy (MBE) on a lithium gallate $\left(\mathrm{LGO}\right.$ or $\left.\mathrm{LiGaO}_{2}\right)$ substrate. The crystal quality of GaN and $\mathrm{InGaN}$ grown on this substrate is significantly improved as a result of a better lattice match between the substrate and the nitride materials $[11,12]$.

In this paper we present the results of confocal photoluminescence (CPL) and timeresolved photoluminescence (TRPL) measurements on two InGaN epilayers. X-ray and CPL confirm the existence of In-segregation in one of the samples. The results of a stretched 
exponential fit to the TRPL intensity decay show the carrier lifetimes is nearly 3 times longer in the segregated sample in comparison to the non-segregated one, in agreement with the predicted decrease in the non-radiative recombination rate associated with increased carrier localization.

\section{EXPERIMENTAL DETAILS}

The two samples used in this study are 500nm thick InGaN epilayers grown by MBE on a LGO substrate on the top of an equally thick $(500 \mathrm{~nm}) \mathrm{GaN}$ buffer layer. One of the samples was intentionally grown to exhibit In-segregation, whereas the other was grown without Insegregation, thus serving as a reference.

CPL spectra were measured using the $400 \mathrm{~nm}$ frequency doubled output beam of a Ti:sapphire laser as a pump. The beam was focused on the sample by a confocal microscope system with a lateral resolution of $200 \mathrm{~nm}$. The PL signal was collected and spectrally analyzed through a fiber attached to a computer-controlled spectrometer equipped with a CCD camera. During the experiment, several PL spectra were collected at different locations on each of the samples.

TRPL measurements were performed using the 200 fs laser pulses produced by a regenerative amplifier (Coherent: RegA) seeded by a Ti:Sapphire laser at 800nm. An optical parametric amplifier (Coherent: OPA) and a frequency doubling BBO crystal provided the upconversion of the RegA pulses to 330nm. The excitation wavelength was chosen such that all the incident photons are absorbed in the nitride layers. The incident beam is focused on the sample by conventional optics with a spot size of $\sim 60 \mu \mathrm{m}$. The back-scattered light from the sample was collected through conventional optics, and spectrally and temporally resolved by a Chromex 250IS monochromator and a Hamamatsu C4334 streakcamera with a typical jitter of 50ps.

\section{RESULTS}

The X-ray diffraction spectra from the two samples under study are presented in figure 1 . The reference sample exhibits three peaks associated with the LGO substrate, the GaN buffer and the InGaN layer. From the difference between the $\mathrm{GaN}$ and $\mathrm{InGaN}$ peaks we estimate the In concentration to be approximately $20.8 \%$, in very good agreement with the expected nominal value. The peak associated with the $\mathrm{In}_{0.208} \mathrm{Ga}_{0.792} \mathrm{~N}$ has a full width half maximum (FWHM) of 289 arc-s, and is broader than the peak corresponding to the GaN layer having a FWHM of 148 arc-s. The broadening indicates there is likely some partial phase separation in the sample and additional dislocations in the InGaN layer not found in the GaN. The spectrum corresponding to the In-segregated sample exhibits two peaks associated with InGaN: one corresponding to a $28.9 \%$ In concentration, and the other to a $44 \%$ In concentration. This is an indication that two completely separate phases are present inside the InGaN layer. We can visualize the sample as $44 \% \mathrm{InGaN}$ "islands" embedded in a $28.9 \% \mathrm{InGaN}$ "matrix". The integrated intensity of the x-ray spectra indicates that the $44 \%$ In phase makes up $15 \%$ of the overall $\mathrm{InGaN}$ material with the rest of the $\mathrm{InGaN}$ attributed to the $28.9 \%$ In phase making the average In composition for the entire film $\sim 31 \%$. The x-ray peak corresponding to the $28.9 \%$ InGaN has a FWHM of 278 arc-s compared to the GaN width of 155 arc-s indicating that, besides the existence of In-rich regions, random alloy fluctuations are also present in the "matrix". The peak corresponding to the $\operatorname{In}_{0.44} \mathrm{Ga}_{0.56} \mathrm{~N}$ is 205 arc-s wide and thus, is likely to have 

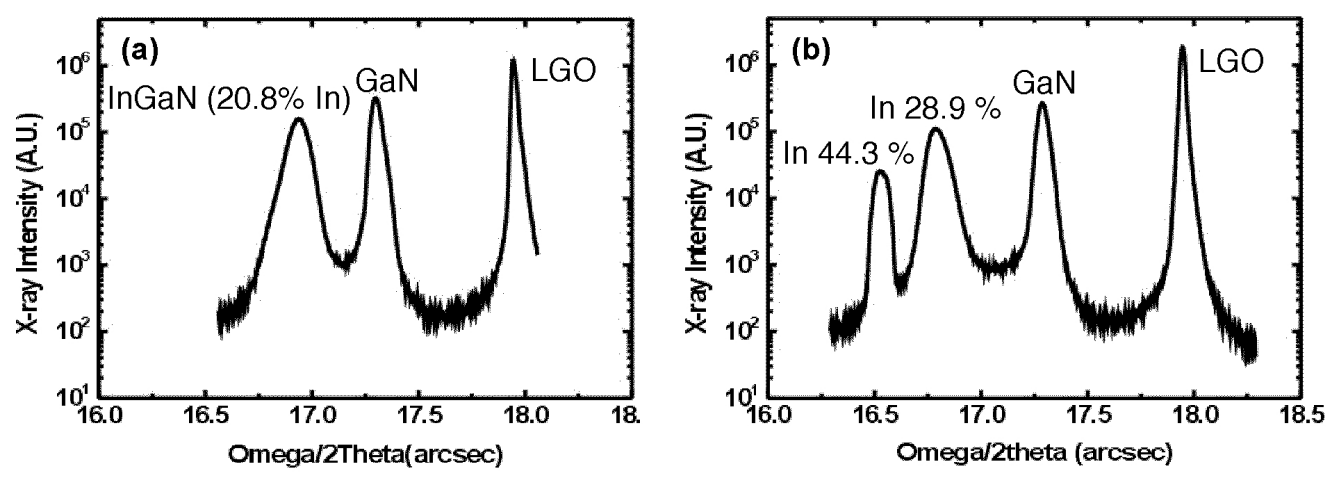

Figure 1. X-ray diffraction spectra for the (a) reference and (b) In-segregated samples. The latter exhibits two peaks indicating the existence of two separate phases.

fewer random alloy fluctuations.

CPL studies confirm the presence of a higher concentration phase inside the segregated sample. Figure 2 presents the PL spectra from several randomly chosen locations across the samples. The dotted lines represent the PL taken using conventional optics. Both samples exhibit very broad $(\mathrm{FWHM}=400 \mathrm{meV})$ PL features as expected for alloys with high concentrations where the presence of random In fluctuations determines the broadening of the PL. The timeintegrated spectrum of the segregated samples shows an overall redshift in comparison to the reference sample suggesting the existence of a higher average In concentration in this sample. The confocal microscope allows us to probe small areas (200nm in diameter) inside the sample and investigate the nature of the redshift observed in conventional PL measurements. Several PL spectra from the reference sample taken at different locations (figure 2(a)) exhibit no significant changes when the beam position is changed on the sample surface. In contrast, the segregated sample PL spectra (figure 2(b)) taken with the same confocal system show significant changes in the PL shape for different beam positions on the sample surface. In fact, there are several peaks, which can be resolved in these spectra. The relative intensity of the peak centered at about $2.5 \mathrm{eV}$ to the one centered at $2.1 \mathrm{eV}$ varies as a function of beam position. These energies match very well to the bandgaps of $\operatorname{In}_{0.289} \mathrm{Ga}_{0.711} \mathrm{~N}(2.5 \mathrm{eV})$ and $\operatorname{In}_{0.44} \mathrm{Ga}_{56} \mathrm{~N}(2.1 \mathrm{eV})$ [13]. The changes in the relative intensity of the two spectral components prove the existence of well-
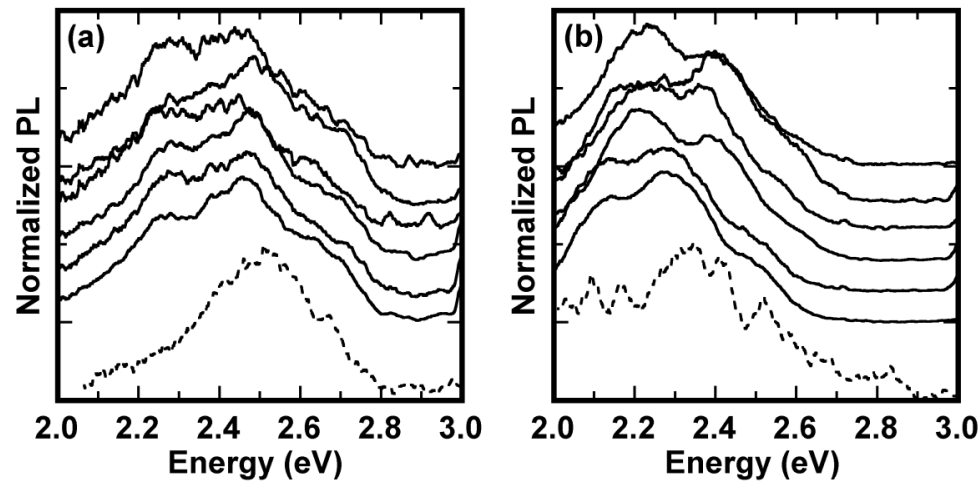

Figure 2. Confocal PL (solid lines) and time integrated PL (dotted lines) spectra of the reference sample (a) and segregated sample (b), taken at room temperature. 
defined areas where the high In concentration component is dominant. This variation shows that the size of the In-rich areas is large (comparable to the beam size - 200nm), and much bigger than any alloy fluctuations existing in the reference sample.

At low temperature, we observed a much stronger signal for TRPL, allowing us to analyze the lifetimes more readily. Figure 3 shows the TRPL spectra from both samples at $15 \mathrm{~K}$. Unlike the spatially resolved measurement, in this experiment, the spot size of the incident beam is much greater than the size of the In-rich regions. Therefore what we observed is the convolution of the PL contribution from several In-rich regions as well as the $28.9 \%$ In "matrix". A red shift is observed in the segregated sample similar to room temperature measurements. The tail emission at $2.1 \mathrm{eV}$ and the dominant feature at $2.5 \mathrm{eV}$ in the segregated sample illustrate the relative abundance of the two phases, mentioned earlier. The PL spectrum is dominated by inhomogeneous broadening due not only to the overlap of PL features associated with the two In phases, but also to the partial phase separation which exists within the $28.9 \%$ In phase. The partial phase separation is also present in the reference sample and as a result, its PL spectrum is characterized by a FWHM comparable to that of the segregated sample. The PL intensity decay appears to be slower in the segregated sample. In an attempt to quantify the difference in PL decay, we plotted the decay of the integrated PL intensity for each sample as shown in figure 4(a). The log (PL intensity) vs. time plot clearly shows that the PL decays for both samples are non-exponential in nature. This is most likely caused by the alloy fluctuation in these samples. Previous studies [14] of PL lifetime have shown that the stretched exponential decay model $[15,16]$ can offer insight into the recombination mechanisms in InGaN-related structures. According to this model the PL intensity decay is described by the following expression:

$$
I(t)=I(0) \exp \left[-\left(\frac{t}{\tau}\right)^{\beta}\right]
$$

based on the assumption that potential fluctuations due to alloy disorder exist inside the sample. The carriers are trapped in the potential minima of these fluctuations. The parameter $\beta$ (the stretched exponential coefficient) represents a measure of the alloy disorder [14].
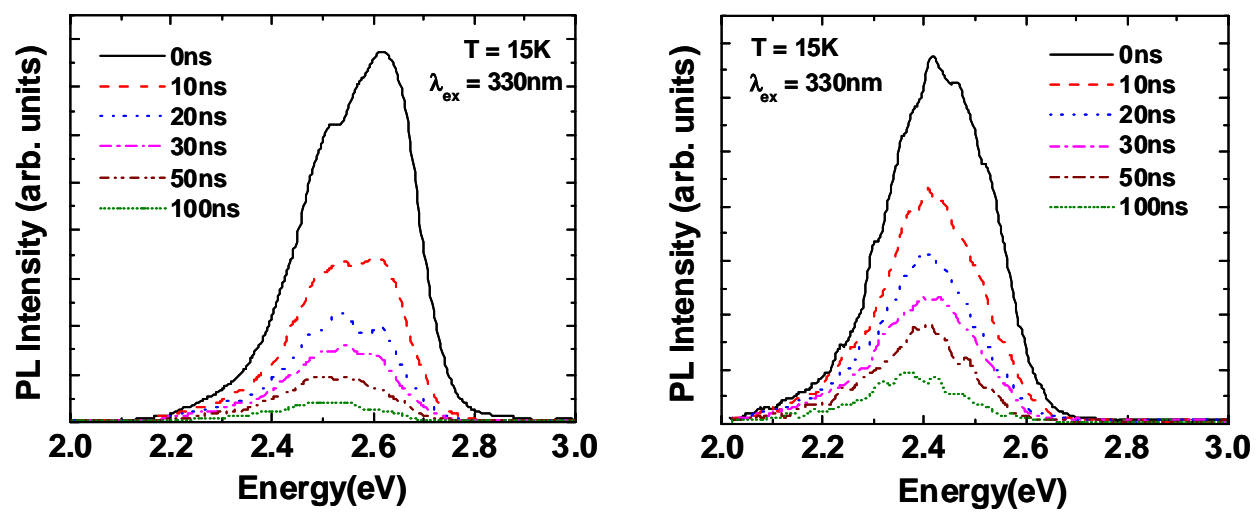

Figure 3. TRPL spectra at different delay times measured at $15 \mathrm{~K}$ for (a) the reference and (b) the segregated sample. The PL spectrum of the segregated sample is redshifted in comparison with the reference sample confirming the presence of a higher indium concentration in this sample 

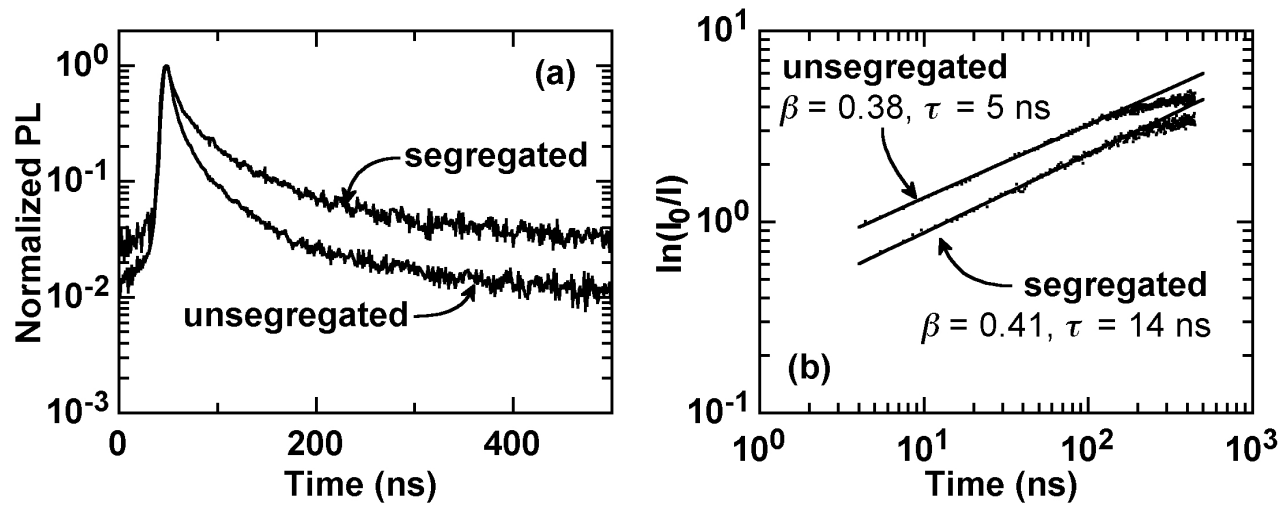

Figure 4. (a) Integrated PL decay taken at $15 \mathrm{~K}$ for the segregated and unsegregated samples and (b) the corresponding $\ln \left(I_{0} / I\right)$ vs. $t$ plots with log-log scales, the solid lines show the fitting for $\beta$ and $\tau$.

In our samples, we fitted the decays with the stretched exponential and obtained the results of $\tau=$ $5 \mathrm{~ns}$ and $\beta=0.38$ for the reference sample, while for the segregated sample, $\tau=14 \mathrm{~ns}$ and $\beta=$ 0.41 . Since the In-rich phase only makes up $15 \%$ of the overall InGaN material in the segregated sample, most carriers are generated in the $28.9 \%$ In region. Subsequent to generation carriers with sufficient energy can relax to the potential minima induced by the phase separation. Therefore, $\beta$ should not be affected by the presence of the In rich regions as long as the $28.9 \%$ phase remains dominant. This accounts for the similarity of the $\beta$ values measured for the two samples. The lifetime that characterizes the segregated sample is nearly 3 times longer than the one measured for the reference sample. Yamaguchi et al. and Narukawa et al. have shown how segregation and carrier localization can have an effect on the PL lifetime [3,6]. In TRPL, the measured emission decay rate is a combination of the rates of radiative and non-radiative recombination, and the PL decay time is related to the radiative and non-radiative lifetimes ( $\tau_{\mathrm{PL}}$, $\tau_{\mathrm{R}}$ and $\left.\tau_{\mathrm{NR}}\right)$ through the following equation:

$$
\frac{1}{\tau_{\mathrm{PL}}}=\frac{1}{\tau_{\mathrm{R}}}+\frac{1}{\tau_{\mathrm{NR}}} .
$$

With In-segregation, the rate of non-radiative recombination is expected to decrease since carriers are now trapped by the In-rich region and confined away from the non-radiative recombination centers. Assuming $\tau_{\mathrm{R}}$ is the similar in both samples then the only explanation for a longer $\tau_{\mathrm{PL}}$ is that $\tau_{\mathrm{NR}}$ has been elongated for the segregated sample. Therefore, the longer lifetime observed in the segregated sample is most likely the result of a reduction in non-radiative recombination rate by carrier localization.

\section{CONCLUSIONS}

LGO serves as a very good substrate for growing strain-free $\mathrm{GaN}$ and $\mathrm{InGaN}$ epilayers in which the effects of dislocations induced by lattice mismatch on the emission efficiency are greatly reduced and the influence of In-segregation on radiative recombination can be studied efficiently. We have presented spatially and time-resolved PL studies of InGaN epilayers grown 
on LGO by MBE. The changes in the spatially resolved CPL spectra as a function of beam position observed in the segregated sample confirmed the existence of In-rich regions indicated by the x-ray measurements on the same sample. The overall redshift of the PL peak energy with respect to that corresponding to a non-segregated samples observed using conventional PL techniques is in agreement with the existence of a high In concentration phase inside the sample. Most importantly, the PL decay times obtained as a result of a stretched exponential fit to the decay data increased by nearly a factor of 3 as a result of In segregation. This observation implies the carriers are no longer trapped by the non-radiative centers but they become localized on radiative states existing in the potential minima created inside the In-rich regions.

\section{ACKNOWLEDGEMENTS}

The authors would like to thank Dr. Haridas E. Pudavar for his assistance in conducting the confocal microscopy on the samples. This research was in part supported by ANC's NSF CAREER Grant \#9733720, ONR YIP Grant \# N00014-00-1-0508, and Doolittle's ONR Grant \#N00014-99-1-0419

\section{REFERENCES}

1. S. Nakamura, and G. Fasol, The Blue Laser Diode, (1997)

2. T. Mukai, D. Morita and S. Nakamura, J. Cryst. Growth 189/190, 778 (1998)

3. A.Yamaguchi, Y. Mochizuki, and M. Mizuta, Jpn. J. Appl. Phys. 39, 2402 (2000)

4. K. P. O'Donnell, R. W. Martin, and P. G. Middleton, Phys. Rev. Lett. 82, 237 (1999)

5. C. A. Tran, R.F. Karlick Jr., M. Schurman, A. Osinsky, V. Merai, Y. Li, I. Eliashevich, M. G. Brown, I. Ferguson, and R. Stall, J. Cryst. Growth 195, 397 (1998)

6. Y. Narukawa, K. Sawada, Y. Kawakami, S. Fujita, S. Fujita, and S. Nakamura, J. Cryst. Growth 189/190, 606 (1998)

7. Y. Narukawa, Y. Kawakami, S. Fujita, S. Fujita, and S. Nakamura, Phys. Rev. B 55, R1938 (1997)

8. O.Ambacher, J. Majewski, C. Miskys, A. Link, M. Hermann, M. Kickoff, M. Stutzmann, F. Bernardini, V. Fiorentini, V. Tilak, B. Schaff, and L. F. Eastman, J. Phys.: Condens. Matter 14, 3399 (2002)

9. S. F. Chichibu, A. C. Abare, M. P. Mack, M. S. Minsky, T. Deguchi, D. Cohen, P. Kozodoy, S. B. Fleisher, S. Keller, J. S. Speck, J. E. Bowers, E. Hu, U. K. Mishra, L. A. Coldren, S. P. DenBaars, K. Wada, T. Sota, and S. Nakamura, Material Science and Engineering B59, 298 (2000)

10. L. Peng, C. Chuang, and L. Lou, Appl. Phys. Lett. 74, (1999)

11. A. S. Brown, and W. A. Doolittle, Applied Surface Science 166, 392 (2000)

12. W. A. Doolittle, S. Kang, and A. Brown, Solid-State Electron 44, 229 (2000)

13. W. Shan, W. Walukiewicz, and E. E. Haller, J. Appl. Phys. 84, 4452 (1998)

14. M. Prophristic, F. H. Long, C. Tran, and I. T. Ferguson, MRS Internet J. Nitride Semicond. Res. 5S1, W11.58 (2000)

15. T. Y. Lin, J. C. Fan, and Y. F. Chen, Semicond. Sci. Technol. 14, 406 (1999)

16. L. Pavesi, J. Appl. Phys. 80, 216 (1996) 